\title{
Vibrational Spectroscopy as a Tool for Studying Drug-Cell Interaction: Could High Throughput Vibrational Spectroscopic Screening Improve Drug Development
}

\author{
Lauren E. Jamieson \\ University of Strathclyde \\ Hugh J. Byrne \\ Technological University Dublin, hugh.byrne@tudublin.ie
}

Follow this and additional works at: https://arrow.tudublin.ie/biophonart

Part of the Medicine and Health Sciences Commons

\section{Recommended Citation \\ This Article is brought to you for free and open access by the DIT Biophotonics and Imaging at ARROW@TU Dublin. It has been accepted for inclusion in Articles by an authorized administrator of ARROW@TU Dublin. For more information, please contact arrow.admin@tudublin.ie, aisling.coyne@tudublin.ie,gerard.connolly@tudublin.ie.}

Jamieson, L.E., Byrne, H.J. (2017). Vibrational spectroscopy as a tool for studying drug-cell interaction: could high throughput vibrational spectroscopic screening improve drug development? Vibrational Spectroscopy, vol. 91, July, pp. 16-30. doi.org/10.1016/j.vibspec.2016.09.003

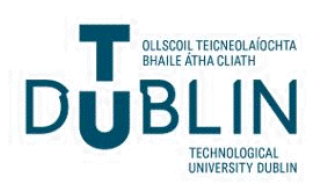




\title{
Vibrational spectroscopy as a tool for studying drug-cell interaction: could high throughput vibrational spectroscopic screening improve drug development?
}

\author{
Lauren E. Jamieson ${ }^{1 *}$, Hugh J. Byrne ${ }^{2}$
}

${ }^{1}$ Centre for Molecular Nanometrology, WestCHEM, Department of Pure and Applied Chemistry, Technology and Innovation Centre, University of Strathclyde, 99 George Street, Glasgow, G1 1RD, United Kingdom, lauren.jamieson@strath.ac.uk.

${ }^{2}$ FOCAS Research Institute, Dublin Institute of Technology, Kevin Street, Dublin 8, Ireland.

\begin{abstract}
Vibrational spectroscopy is currently widely explored as a tool in biomedical applications. An area at the forefront of this field is the use of vibrational spectroscopy for disease diagnosis, ultimately aiming towards spectral pathology. However, while this field shows promising results, moving this technique into the clinic faces the challenges of widespread clinical trials and legislative approval. While spectral pathology has received a lot of attention, there are many other biomedical applications of vibrational spectroscopy, which could potentially be translated to applications with greater ease. A particularly promising application is the use of vibrational spectroscopic techniques to study the interaction of drugs with cells. Many studies have demonstrated the ability to detect biochemical changes in cells in response to drug application, using both infrared and Raman spectroscopy. This has shown potential for use in high throughput screening (HTS) applications, for screening of efficacy and mode of action of potential drug candidates, to speed up the drug discovery process. HTS is still a relatively new and growing area of research and, therefore, there is more potential for new techniques to move into and shape this field. Vibrational spectroscopic techniques come with many benefits over the techniques used currently in HTS, primarily based on fluorescence assays to detect specific binding interactions or phenotypes. They are label free, and an infrared or Raman spectrum provides a wealth of biochemical information, and therefore could reveal not only information about a specific interaction, but about how the overall biochemistry of a cell changes in response to application of a drug candidate. Therefore, drug mode of action could be elucidated. This review will investigate the potential for vibrational spectroscopy, particularly FTIR and Raman spectroscopy, to benefit the field of HTS and improve the drug development process. In addition to FTIR and Raman spectroscopy, surface enhanced Raman spectroscopy (SERS), coherent anti-Stokes Raman spectroscopy (CARS) and stimulated Raman spectroscopy (SRS), will be investigated as an alternative tool in the HTS process.
\end{abstract}

Keywords: FTIR, Raman, SERS, nonlinear Raman, drug screening, HTS

\section{Introduction}

Vibrational spectroscopy has gained momentum as an important tool for biomedical applications. Vibrational spectroscopy encompasses several techniques, most significantly, infrared (IR) spectroscopy and Raman spectroscopy, which detect bond vibrations. These techniques have been applied to biological samples, including tissue in vivo[1], excised tissue ex vivo, tissue sections ex vivo[2], blood[3] and serum[4] ex vivo, and single cells[5] in vitro, in which signals from biomolecules such as proteins, lipids and DNA/RNA are observed and used to characterise samples.[6] In particular, the application of IR and Raman spectroscopy for label free disease diagnosis is being extensively studied in the biomedical vibrational spectroscopy field. Extensive research has been invested into the potential for increased objectivity and automation in histopathology and cytology with a movement towards 
spectral pathology as the ultimate goal.[6] Standard clinical practice requires analysis of haematoxylin \& eosin (H\&E) stained tissue sections by a pathologist for disease diagnosis. Attempts are being made to move towards more automated disease diagnosis in tissue sections, and cytology samples, using vibrational spectroscopy. One of the major advantages of IR and Raman spectroscopy over, for example, histology, fluorescence staining and immunohistochemistry, is that it is a label free method, detecting native biochemical markers of cells and tissue via characteristic bond vibrations, and associating changes in them with disease onset and progression, rather than the morphological changes which characterise the disease.[6]

In 2014, the Clinical Infrared and Raman Spectroscopy for Medical Diagnosis (CLIRSPEC) Network, funded by UK EPSRC, was set up with the goal of accelerating the translation of spectroscopy into the clinic.[7] Raman4Clinics is a similar European network (EU Cost Action BM1401), specifically established to promote the translation of Raman spectroscopy into clinical applications.[8] Raman spectroscopy has already been applied for clinical use in vivo, with a particularly exciting recent development, in which Raman spectroscopy was used in real time for assessment of tumour margins for determining tissue to be excised in a brain tumour operation, being featured on the BBC news.[9,10] IR spectroscopy is also moving towards clinical applications, a particularly exciting clinical trial is currently active in Thailand, demonstrating the use of attenuated total refection Fourier transform IR (ATRFTIR) spectroscopy for detection of malaria in blood samples using minimal sample preparation, allowing for quick and easy detection with portable devices and no need for scientist input.[3,11]

While there is an intensive drive towards moving vibrational spectroscopy techniques into the clinic for diagnostic purpose, there is also huge scope for the use of vibrational spectroscopy in other biomedical research areas. There are many challenges facing translating vibrational applications into the clinic. These include the extensive ethical considerations and approval required for in vivo and ex vivo use, and the resistance to change among pathologists who have been following the same established protocols for an extensive time.[6] Another area where there is potential for the use of vibrational spectroscopy for biomedical application is as a tool in drug development; pre-clinical screening drug candidates to select successful 'hits' to take forward into clinical trials. As this generally involves in vitro models and is itself a more recent and growing area of development, there is potential to have a larger impact in shaping the research within this field. There is huge legislative pressure on the development of in vitro methods to replace animal testing, making in vitro based drug testing platforms of significant importance (Directive 2010/63/EU on the protection of animals used for scientific purposes[12]; United States the $106^{\text {th }}$ Congress - Public Law $106-545$ - Dec 19, 2000[13]). Currently, high throughput screening (HTS) methods for detecting successful lead drug candidates are highly dependent on fluorescence techniques for assessment of efficacy.[14] The assays used tend to probe specific binding interactions between a drug and protein, reporting in a univariate manner via fluorescence. These techniques require assays to be available for each specific interaction or phenotype; probing just one interaction and not the additional effects a drug may have. The cellular samples may also be subject to labeling or photobleaching and are destructive techniques as a result.

Both IR and Raman spectroscopy have been used to assess the effects of drugs on cells, with potential to measure changes in a high throughput manner and therefore be used in drug screening.[15,16] These techniques could provide advantages over standard high throughput fluorescence based methods by revealing increased biochemical information using a label free method. They detect detailed biochemical information in a more quantitative manner, with 
the potential to not only pick up a change in response to drug treatment, but to reveal detailed biochemical information on how drugs may alter cellular biochemistry and reveal a potential 'mode of action'.[17]

In addition to traditional IR and Raman spectroscopic methods, there are more advanced vibrational techniques that can be employed for alternative high throughput screening assays. Surface enhanced Raman spectroscopy (SERS), has been used to develop assays for various applications with many benefits over fluorescent assays.[18] These include lack of photobleaching, ability to multiplex and probe multiple interactions in one assay, and increased ability for quantification of measurements. SERS can also be used as a means of simply enhancing Raman signals, considerably reducing acquisition times in Raman analysis.[19] Coherent anti-Stokes Raman spectroscopy (CARS) and stimulated Raman spectroscopy (SRS) may lead the future for spectroscopic HTS. They are nonlinear Raman techniques, which overcome the inherent weakness of spontaneous Raman spectroscopy.[20] Therefore, measurements can be performed faster, a vital benefit for HTS.

This review will therefore focus on the potential of vibrational spectroscopy for use in analysing the interaction of drugs with cells and, in particular, how these techniques could potentially be employed for the screening of drug candidates in a high throughput manner, a label free method providing a wealth of biochemical information that could reveal important information regarding the interaction of drug candidates with cells. Ultimately these HTS techniques could be used for companion diagnostics and personalised medicine, assessing suitability of a particular therapy for a particular patient or patient group.

\section{Current high throughput screening techniques: could vibrational spectroscopic screening improve these?}

High throughput screening (HTS) is widely used in the pharmaceutical industry for faster identification of drug 'hits', compounds exhibiting a desired biological response to a potential drug compound.[21] Often, this biological response involves a binding event between a compound and a particular protein target or DNA[22], and this is typically monitored using a fluorescence assay based technique. HTS aids drug discovery by speeding up the process of identifying compounds, from large compound libraries, with the desired mode of action. Development of a new drug product from start to end is a very long process, taking on average 12 years, and costing, on average \$1.2 billion per product.[23] Moving from low throughput techniques at the initial stages of drug development, whereby compounds are screened one at a time in a highly manual intensive manner, to highly automated high throughput screening techniques allowing multiple compounds to be screened simultaneously, can help minimise the time for the drug development process. To compare, HTS allows ca. 10,000 compounds, and even up to 100,000 compounds, to be screened in one day, compared to a maximum of $c a .500$ compounds using low throughput screening techniques.[21] Initial compound screening generally identifies compounds that exhibit the desired activity. For successful compounds, modified versions can then be developed using synthetic techniques, producing new libraries of compounds to be screened. It is also necessary to consider optimal concentration of 'hit' compounds, any negative side effects they may have and their toxicity. Therefore, it is appropriate to employ screening techniques at various stages in the drug discovery process. HTS allows many more compounds to be screened in one day, and could easily reduce a process that could take 1 year to screen using low throughput methods, to only 1 day using HTS. 
The key characteristics of HTS techniques are: simple, fast, cheap and efficient. Compounds are typically screened in 96-, 384- or 1536- well plates, ideally using minimal volumes.[14] The most recent developments using microfluidics have allowed even smaller volumes to be used with increased throughput capability.[24] In order to understand the potential benefits of vibrational spectroscopic techniques in HTS, over current techniques, it is important to understand the principle behind current techniques along with their limitations.

Vibrational spectroscopic screening is being considered as an alternative and improved technique in screening potential drug compounds for efficacy; potentially in conjunction with other factors such as mode of action and toxicity in a high throughput manner. Primarily, this review will focus on in vitro cell based assays for compound screening. In HTS this is one of the two main assay types (the other being biochemical assays). In a biochemical assay, compounds are screened for action on their particular target in their purified form.[14] Biochemical assays can be heterogeneous or homogenous, heterogeneous assays requiring multiple steps, compared to simpler homogeneous assays that are simply 'mix and read' type systems. Detection techniques in HTS are predominantly fluorescence and luminescence based techniques, including standard fluorescence and luminescence assays as well as extensions of these such as fluorescence resonance energy transfer (FRET)[25], fluorescence polarization (FP)[26], homogeneous time resolved fluorescence (HTRF)[27] and fluorescence correlation spectroscopy (FCS)[28]. Assays are developed to detect specific binding processes or to probe particular pathways, networks or phenotypes.[29] Some of the most common targets are G-protein coupled receptors, enzymes, hormones, ion-channels, nuclear receptors and DNA.[14] Both fluorescence and luminescence detect photons, with some common assays using luciferase reporters[30], green fluorescent proteins[31], $\beta$-galactoside[32] and $\beta$ lactamase[33].[29] Common fluorescent reagents for cytotoxicity screening are Alamar blue[34], MTT (3-[4,5-dimethylthiazol-2-yl]-2,5-diphenyl tetrazolium bromide)[34] and sulforhodamine $\mathrm{B}[35]$.

To answer the question 'could high throughput vibrational spectroscopic screening improve drug development?', it is necessary to consider whether it can meet the basic needs of HTS by being simple, fast, cheap and efficient, and, ultimately, whether it can supersede the current techniques in doing so. Standard HTS assays often monitor only one interaction, giving a 'on' or 'off' response typically in reaction to a binding event, at best giving a graded readout, which would provide quantitative information also on this single response. Vibrational spectroscopy can provide an 'on' or 'off' response also, in terms of overall changes in the spectrum in response to drug application, but could also be used to reveal a whole array of additional information; how a drug interacts with a cell, possible toxic side effects of the drug, how a drug effects the biochemical make up of a cell in terms of e.g. protein/lipid/DNA concentration. This may spare the need for additional assays, increasing speed. In addition, another benefit is the label-free aspect of the vibrational techniques, which both decreases additional cost for fluorescent reagents, and makes the assay process simpler, faster and more efficient by decreasing additional steps involved in adding reagents for detection. While the addition of spectroscopic instruments may increase cost of screening, high throughput spectrometers are already on the market and costs will decrease as these become more popular.[36]

While the focus is on detection of drug interaction in cell-based assays via vibrational spectroscopy, there is also potential for applications in biochemical assays with isolated cell components. In particular, the technique of surface enhanced Raman spectroscopy (SERS) has been used extensively in assay development in other biomedical research areas for many applications, including ELISA type immunoassays.[37] It has many benefits over standard 
fluorescence based assays, including lack of photobleaching, multiplexing ability[38] and often increased sensitivity. SERS has also been applied to in vitro cellular assays, to analyse, for example, enzyme activity.[18] Therefore, while SERS does not have the label-free advantage of native vibrational spectroscopic techniques, it provides many benefits over traditional fluorescence techniques with potential to increase speed through, for example, multiplexing capabilities.

\section{Data analysis: interpreting spectroscopic data}

One of the major benefits of vibrational spectroscopy over fluorescence is the wealth of information that can be obtained. An individual spectrum acquired using IR or Raman spectroscopy contains the signatures of the many constituent biomolecules, over many data points, and therefore data analysis is carried out in a multivariate manner, to elucidate the wealth of information on how a specific drug candidate affects a cell. However, with this comes the requirement for more complicated data analysis protocols.

In this section, some of the major considerations to be made for interpretation of spectroscopic data in terms of pre-processing and post-processing analysis techniques will be explored. Byrne et al.[39] recently summarised the major pre and post processing methods employed for IR and Raman spectroscopic analysis of tissues and cells. Pre processing methods involve improving spectral quality by removal of background features caused by physical effects, sample preparation and substrate. This helps remove the effects of instrument-to-instrument and sample-to-sample variation, therefore standardising the technique for translation into clinical and HTS applications. Particular pre processing considerations for IR spectroscopy involve applying Mie scattering correction algorithms[40] and water correction algorithms[41] for aqueous samples (see section 4 for a full discussion). A major consideration for both IR and Raman spectroscopy is substrate, with different substrates creating different background contributions in the spectra.[42] Due to difficulties removing all background contributions experimentally, algorithms, going from simple straight or polynomial line subtractions to more complicated algorithms, such as extended multiplicative scatter correction (EMSC)[43] and the "rubber band" model, are often applied to spectra before further data processing. Additional commonly employed pre processing steps include smoothing, truncation, normalization, taking spectral derivatives and cosmic ray removal (Raman).[44]

Moving to post processing methods, the large volume of information obtained from vibrational spectroscopy, requires multivariate data analysis to extract information, in this case regarding how a drug interacts with and alters a cell. In fluorescence spectroscopy, typically only one parameter is measured, i.e. fluorescence at a specific wavelength. Most fluorescence based techniques work via a simple 'on/off' mechanism whereby fluorescence is switched 'on' or 'off' in response to the biological event being probed. At best, this can extend to a univariate model, giving quantitative information by measuring the absolute fluorescence, and comparing it to calibration data. In comparison, a single IR or Raman spectrum can have hundreds or thousands of data points, recording multiple wavelengths in one spectrum, revealing detailed quantitative information regarding subtle changes in cellular biochemistry. Signals corresponding to particular bond vibrations are observed in spectra and each reports somewhat quantitatively via signal intensity on biochemical composition. This can reveal detailed metabolic information. Therefore, subtle changes in metabolism in cells can potentially be picked up using vibrational spectroscopic techniques, and the mass of information available in a single spectrum allows potential 'mode of action' to be determined, in addition to efficacy. It is possible to consider only one or two specific 
wavenumbers when analysing this data, therefore simplifying to univariate or bivariate data. However, this fails to consider the volume of additional information that could provide additional discriminatory power. Therefore, multivariate models have been developed to consider the large number of parameters contained within a hyperdimensional dataset.

While multivariate analysis can provide additional discriminatory power, these require more complex data processing that may take a longer time and require more expensive software. Recent developments in IR spectroscopy have looked at discrete frequency imaging using, for example, quantum cascade lasers (QCL)[45], where spectra are acquired over only small wavenumber ranges. These techniques have the advantage of speed, if it is possible to discriminate between conditions using a narrower spectral range than standard range of FTIR spectroscopy. Some of the most common multivariate models are principle component analysis (PCA) and partial least squares regression analysis (PLSR), which look for the largest variances in the data set in an unsupervised and supervised manner respectively. Cluster analysis is, perhaps, the simplest multivariate analysis technique. It groups spectra according to similarity, with similar spectra being put in one 'cluster'. For drug-cell studies hierarchical cluster analysis[46] is the most widely applied cluster analysis method.

PCA is the most widely used data analysis technique in this area of research. It is used to reduce the data dimensionality by creating new variables, principle components, to describe the maximum variance in the data set. Principle component one will describe the maximum variance followed by principle component two for the next most variance, and so on. This technique can, for example, distinguish between two sets of conditions such as with and without drug, and the principle component loadings will show the major variances in spectral features that differ between conditions.[47] By assigning these differences to particular biomolecule vibrations, insight can be gained into how and where a drug is interacting with cells. Another commonly used data analysis technique is PLSR. PLSR could have important application, in particular, to the study of cells using vibrational spectroscopy, as it is used to build up a predictive model on how spectral signature changes in response to a systematic change in conditions. Nawaz et al.[48] performed a particularly interesting study where they highlighted the ability of PLSR to distinguish changes in spectral signals resulting from chemical binding of the drug cisplatin to DNA, and the subsequent physiological changes this induced. By building up a database of spectral responses from drugs of known mechanism, this could serve as a reference for inferring 'mode of action' for new drugs during HTS screening application. Therefore, PLSR could prove to be a powerful technique for HTS application. In addition to cluster analysis, PCA and PLSR, there are numerous other data analysis techniques being employed in the field of biomedical spectroscopy. These include vertex component analysis[49], classic least squares analysis[50], spectral cross correlation analysis[50], artificial neural networks[51] and genetic algorithm[52], but for the purpose of this review these will not be discussed further. For further discussion of these techniques and their application, reference is made to Byrne et al.[39].

It is worth noting also that while vibrational spectroscopy reveals a wealth of information in one spectrum, it can only really be considered semi-quantitative. This is a result of macromolecular bond vibration overlap in spectra due to the large volume of signals from and therefore peaks tend to have contributions from a number of species.[53] Therefore, while analysis of changing intensity of individual bands could provide quantitative information regarding subtle changes in biochemical composition, care must be taken as often peaks have underlying contributions from other species contributing to absolute intensity. 
To conclude this section, fluorescence assays are standardly developed to monitor one specific drug-protein interaction targeted by the drug. While vibrational spectroscopic techniques could potentially monitor spectral changes in response to such a drug-protein interaction, more detailed information can be gained, potentially revealing additional interactions that a particular drug may have on the metabolism of the cell. Therefore, there is potential for a very detailed picture of drug-cell interaction to be gained through vibrational spectroscopic analysis. One 'assay' using vibrational spectroscopy could potentially be used to screen drugs with multiple and different modes of action in one step. Using fluorescence this would require multiple screening steps with different assays to report on each potential interaction, and would depend upon having a good knowledge of the potential interactions, and an assay available to probe that. While drugs are commonly designed with a particular drug-protein interaction in mind, opening up the possibility of screening for multiple 'modes of action' could provide many benefits. Detailed biochemical information is gained, and this could also screen for efficacy and toxicity simultaneously. In order to provide this detailed information on drug-cell interaction, however, vibrational spectra must first be subjected to pre and post processing data analysis to extract the subtle changes in biochemistry using multivariate techniques.

\section{Infrared spectroscopy for monitoring drug-cell interaction}

There has been extensive research using IR spectroscopy, performed in vitro, to monitor the cellular response to drugs. Much of this research has focused on cancer cells and monitoring the changes in the spectral signatures of cells in response to anticancer agents. In 2015, Hughes et al.[15] highlighted, in a short review article, the potential for IR microspectroscopy to be used as a label free screening platform - "Preclinical screening of anticancer drugs using infrared (IR) microspectroscopy".

Many studies have reported the effects of anticancer agents, including polyphenols[54], cardiotonic steroids[55], and platinum compounds[56,57], on cancer cells using IR spectroscopy. Typically, IR spectroscopy uses Fourier transform (FTIR), to allow fast acquisition of data over a wide spectral range. There are three primary measuring methods used in FTIR spectroscopy - transmission, transflection and attenuated total reflection (ATR). It is important to consider the advantages and disadvantages of these techniques in terms of speed, cost and sample preparation if techniques are ultimately to be translated to a HTS platform. All three methods have been used to investigate drug-cell interactions. The primary disadvantage of transmission IR is that it generally requires high cost substrates such as calcium fluoride, which are also brittle, and therefore care must be taken when handling them.[39] The cost of the substrate would make translating this technique into a HTS platform difficult as a result of the cost involved. However, if substrates could be reused this would reduce the consumable cost. Transflection IR, in comparison, uses cheap MirrIR slides that are easier to handle.[39] As the beam passes through the sample twice, transflection IR also results in better signal-to-noise, and a reduced acquisition time can be used to achieve the same quality spectra as with transmission IR.[58] Alternatively, this means for transflection IR can use samples with less thickness can be used. The major downfall of transflection IR, however, is the presence of the "Electric Field Standing Wave" effect, with can affect the resulting absorbance profile, making quantitative measurements inaccurate.[58] Finally, ATR IR gives the best signal-to-noise, and is therefore the fastest technique.[58] It also requires little sample preparation, however varying force applied to the sample can affect the signals in the spectra and therefore correction algorithms are often required.[59] The pressure applied also has the potential to damage the sample.[58] 
Another important consideration in FTIR spectroscopy, is that historically synchrotron radiation was commonly used to obtain sufficient signal to noise for high spatial resolution imaging. While FTIR spectroscopic studies on biological samples were previously highly dependent on synchrotron radiation sources due to diffraction limitations using a globar source limiting spatial resolution[60], recent advances have expanded the potential of benchtop instruments to achieve desirable resolutions[61]. It also may not be necessary to achieve high spatial resolutions for HTS techniques, instead taking spectra from a population of cells, representative of the sample. Draux et al.[62] demonstrated that FTIR spectral response of Calu- 1 lung cancer cells, to the drug gemcitabine, was similar between single cell measurements using synchrotron radiation FTIR (SR-FTIR) and cell population measurements using a benchtop instrument in transmission mode with a HTS-XT (high throughput screening eXTension) attachment. Inhibition of proliferation upon drug treatment was detected, provided the growth inhibition was above $25 \%$, and a concentration dependent response was observed. In this example, hierarchical cluster analysis was used for data analysis, one of the simplest multivariate data analysis techniques.

One of the major downfalls of IR spectroscopy is that water is strongly IR active. Therefore, in aqueous biological samples, a lot of information is 'swamped' by the strong water vibration signals observed at $c a .3300 \mathrm{~cm}^{-1}$, and $c a .1650 \mathrm{~cm}^{-1}$. Notably, the band at $c a .1650 \mathrm{~cm}^{-1}$ appears in the region of particular interest for biological samples, overlapping with the protein amideI band at $c a .1655 \mathrm{~cm}^{-1}$. This is ultimately a problem when moving towards live cell analysis in vitro and many of the cell-drug interaction studies have been carried out on fixed cells. Recent developments using microfluidic style devices have allowed for better live cell analysis, and could be vital to move this to a HTS platform.[58] Microfluidic devices tend to use minimal volumes, which is an added advantage for HTS considerations. Even when the aqueous environment of the cells is removed, contributions from water vapor, as well as $\mathrm{CO}_{2}$, in the atmosphere can affect spectra.[41] Much of this water and $\mathrm{CO}_{2}$ vapor contribution can be removed by purging instruments. It is likely, however, that water contributions will still be observed, and water correction algorithms need to be employed to remove these. These algorithms could also allow cells in live aqueous environment to be investigated successfully. Therefore, this drawback no longer limits the use of IR spectroscopy, but makes data analysis protocols more complicated to allow for correction algorithms to be developed and employed.

Another major consideration in IR spectroscopy is Mie scattering, a physical scattering effect caused by the wavelength of light being close to the size of object being probed, i.e. cells and subcellular organelles. In addition to adding a broad, undulating background to the spectra, resonant effects can cause significant distortions of the characteristic spectral features. Again, correction algorithms have been developed to remove the effects of Mie scattering. However, correction algorithms are not perfect and attempts are constantly being made to improve these to better correct for Mie scattering.[40] In particular, when considering monitoring spectral changes in response to potential drug compounds, it is important that Mie scattering contributions are considered. This is particularly relevant in this application, as drugs will often cause morphological, as well as chemical changes to cells. Morphological changes will potentially lead to changes in Mie scattering contribution and therefore this, and not chemical changes, could be the cause of spectral changes. Therefore, it is important to understand and eliminate the effects of Mie scattering when considering chemical changes in response to drug candidates. There is particular relevance of this effect when considering the cell nucleus, which is often the cellular region with most Mie scattering, and is also often subject to significant morphological changes in response to drugs that cause apoptosis. Drugs often work via induction of apoptosis, which commonly causes nuclear condensation and 
fragmentation, which could have a significant effect on Mie scattering contribution. While these contributions complicate IR spectroscopic measurements, it should be noted that they also provide additional information regarding the physical characteristics of cells i.e. morphology, which could potentially be used in discrimination. For example, this could potentially discriminate normal from apoptotic cells due to morphological changes, in particular, from the cell nucleus. Therefore, both physical and chemical information could be gained from IR spectroscopy, providing that contributions can be deconvoluted successfully, providing a wealth of information for spectroscopic discrimination.

Another important point for consideration in IR, and also Raman, spectroscopy, which has been addressed in various studies, and which would be applicable to all techniques looking at cell-drug interactions, is cell cycle. It is important to consider spectral differences between cells at different point in cell cycle, to ensure that spectral responses observed upon drug treatment are due to the drug and not cell cycle differences.[63] If IR spectroscopy is capable of distinguishing cell cycle in conjunction with response to drug compound, this provides information not accessible using simple fluorescent techniques by which cell cycle is not monitored. Initial studies into the effect of cell cycle on drug response used complimentary fluorescence staining techniques to ascertain and separate cells by cell cycle.[63,64] Hughes et al.[63] used the dye propidium iodide (PI), which binds in a quantitative manner to doublestranded DNA, combined with fluorescence-activated cell sorting (FACS) to separate cells into $G_{1}$ (Gap 1), S/G2 (synthesis/post DNA synthesis) and M (mitosis) phases. In this study, the effect of the drug 5 -fluorouracil (5FU), known to disrupt $S$ phase, and three novel goldbased chemotherapeutic compounds (KF0101 (KF1), KF0113 and KF0501), on A-498 renal carcinoma cells, was investigated using SR-FTIR. Cells were treated with each chemotherapeutic agent for $24 \mathrm{hrs}$ and $72 \mathrm{hrs}$, and untreated and treated cells were fixed before staining with PI and FACS sorting to separate cells into cell cycle phase. Results highlighted the significance of separating cells by cell cycle, with discrimination by PCA between untreated, 5FU treated and KF1 treated observed for S phase cells but not the full dataset. This is consistent with the known action of 5FU. Some of the important considerations already highlighted for IR spectroscopy were also highlighted in this study, particularly the importance of performing Mie scattering correction. Significant differences in Mie scattering contribution between the different cell cycle stages were observed, likely due to changes in amount of cellular content[65], and therefore the RMieS-EMSC correction algorithm[40] was used. The ability to detect changes after $24 \mathrm{hr}$ treatment was highlighted as an advantage with respect to HTS, and the benefit of distinguishing cell cycle without additional staining was confirmed as an ideal next step. In addition, this particular study was carried out on fixed cells and using a synchrotron radiation source. A similar study was carried out by Derenne et al.[64] looking at cell cycle effects on PC-3 prostate cancer cells in response to anticancer drugs, using a benchtop instrument. This study looked at the difference between $\mathrm{G}_{0} / \mathrm{G}_{1}$ and $\mathrm{G}_{2} / \mathrm{M}$ phase cells both untreated and treated with paclitaxel, an anticancer drug that stops microtubule depolymerisation in the $\mathrm{M}$ phase of the cell cycle. Again this study relied on fluorescence data to assign cell cycle stage, and differences in the FTIR spectra between cell cycle stages were investigated. The primary conclusion, however, was that the FTIR spectral signatures did not show large variations between cell cycle stage, in comparison to the differences observed upon drug treatment.

In a more recent study from Jimenez-Hernandez et al.[66] (Figure 1), a robust support vector machine (SVM) was employed to discriminate FTIR spectral signatures for different cell cycle stages, previously deemed "small" by Derenne et al.[64], with $83.7 \%$ average accuracy. While these spectral differences may be small, the ability to discriminate cell cycle stages using FTIR, allows more detailed and accurate analysis of drug effects. As well as removing the 
possibility of cell cycle stage differences confounding drug response results, this could provide a platform for determining 'mode of action' of a drug, through knowledge of what cell cycle stage is most affected by the drug. Importantly, this study highlighted that, for Caki-2 renal cell carcinoma cells treated with 5FU and paclitaxel for $24 \mathrm{~h}$, the primary FTIR spectral signature changes could be attributed to apoptosis. These changes relate to lipid changes and reduced protein, previously observed as characteristics of apoptosis observed in FTIR spectra.[67,68] While, observation of a distinguishable apoptotic response could still prove useful as a marker in HTS technologies, ability to extract more detailed spectroscopic information regarding 'mode of action' shorter drug exposure time may be required.

It is not only cell cycle stages that could confound FTIR spectral analysis of cells responding to drug treatment. Rutter et al.[69] investigated the difference in response of gemcitabinesensitive and gemcitabine-resistant CALU-1 epidermoid lung cancer cells to gemcitabine. By cloning sensitive and resistant cell populations, from a mixed population, they showed that PCA did not discriminate treated from untreated resistant cells, but did for sensitive cells. While, obtaining multiple spectra over a cell population growing in, for example, a well in a 96-well plate, may be enough to average effects such as cell cycle contribution and resistance/sensitivity, it would be more accurate to take these characteristics into consideration. FTIR spectroscopy has the potential to provide spectral signatures that allow these multiple characteristics to be analysed in addition to drug response.

\section{Raman spectroscopy for monitoring drug-cell interaction}

The potential of IR spectroscopy to monitor drug-cell interactions and potentially translate into a HTS platform for drug screening has now been explored extensively. There has been similar research into drug-cell interaction using Raman spectroscopy. The main downfall of Raman in comparison to IR spectroscopy is low signal intensity, and therefore longer acquisition times required to achieve sufficient spectral information. As speed is one of the primary characteristics desirable for HTS, this is a significant hurdle for Raman to be employed in such a system. However, Raman spectroscopy also exhibits many advantages in comparison to IR spectroscopy. The main advantage of Raman spectroscopy is that high spatial resolutions in a confocal microscopic geometry are possible due to the use of optical wavelengths.[70] It is a scattering technique, allowing any ultraviolet (UV), visible or IR wavelength to be used. While UV wavelengths allow higher spatial resolutions and scattering efficiencies to be achieved, there is increased susceptibility to photodamage and fluorescence from resonant cellular chromophores at these wavelengths. Therefore, near IR (NIR) is often the wavelength of choice for Raman spectroscopy to achieve an optimal balance of spatial resolution along with limited photodamage and fluorescence background.

In addition, water is only very weakly Raman active, and therefore aqueous samples can be analysed easily, without the need for complicated algorithms for water correction. Similarly, Mie scattering is not an issue in Raman spectroscopy, again negating the need for complex computer algorithms for spectral adjustment. Raman spectroscopy is compatible with samples in almost any state, while IR spectroscopy requires specific and more complicated sample preparation steps when used in transmission and transflection mode. The one particular consideration that must be made in terms of sample preparation for Raman spectroscopy, is choice of sample substrate.[42] Plastic and glass both give background signals in Raman spectra, meaning that often cell samples are plated on substrates such as $\mathrm{CaF}_{2}, \mathrm{BaF}_{2}$ or sapphire windows with minimal background.[42] For a HTS platform this is an important consideration in terms of cost, as these background free substrates are more expensive. Glass can be used as a Raman substrate with minimal background contribution, 
particularly at certain wavelengths. Therefore, while there is less background using the more expensive substrates, where cost is important, it is possible to use glass. It is also worth considering more intricate substrate platforms, particularly in the context of HTS, such as microarrays. Buckmaster et al.[71] report on the use of a gold-patterned silicon oxide substrate, for attachment of single-cells, and subsequent Raman analysis. Here, the response of single DAOY human medulloblastoma cells to the chemotherapeutic agent etoposide was investigated in real time at various time points. Raman spectra revealed detailed biochemical information, and were able to detect apoptotic changes in single cells in response to drug treatment. This study also found that a small number of cells showed resistance, and therefore was able to discriminate varied responses within a cell population, by performing single cell analysis on this microarray. The changes in intensity of individual peaks corresponding to particular bond vibrations were investigated, and thus considered data in a univariate manner. Similar to IR spectroscopy, however, Raman analysis provides a wealth of information and therefore multivariate data analysis techniques are often employed for spectral analysis.

Raman spectroscopic analysis of drug-cell interactions has focused both on changes in the cellular Raman spectra to drug application, and also to intracellular tracking of the drug and its metabolites. Therefore, a wealth of information can be gained using Raman spectroscopic analysis. Subcellular resolution can be achieved due to the high spatial resolution achievable, allowing intracellular organelles to be resolved, often using cluster analysis, an unsupervised multivariate data analysis technique. Raman spectra are typically recorded at higher spectral resolution than IR spectra, and therefore more detailed biochemical information can be gained, with more potential for elucidating 'modes of action' by analysis of the detailed spectral changes. El-Mashtoly et al.[46] demonstrated the capability of Raman spectroscopy for monitoring distribution of erlotinib, and its metabolic products in colorectal adenocarcinoma SW480 cells. Insight into the subcellular localisation and metabolic products of erlotinib was revealed, hierarchical cluster analysis allowing subcellular resolution in a non invasive and label free manner, and allowing intracellular location of erlotinib and its metabolic product to be determined.

Farhane et al.[72] took this a step further, using Raman spectroscopy to determine not only subcellular location of delivered drug, but also the effects the drug has on cellular Raman signature (Figure 2). This thorough study investigated the uptake of doxorubicin to A549 human lung adenocarcinoma cells. Raman microspectroscopy confirmed fluorescence results, indicating that doxorubicin was localised to the nucleus after $24 \mathrm{hr}$ exposure, as doxorubicin peaks were only observed in spectra from the nucleus and nucleolus, and not the cytoplasm. In addition to subcellular localisation of doxorubicin, PCA was employed to distinguish cellular biochemical changes in each distinct cellular region in response to drug exposure, and elucidate the mechanism of drug action. A further study by Farhane et al.[73] demonstrated that Raman spectroscopy could be employed to differentiate the respective responses of two different lung cancer lines (A549 and Calu I) to doxorubicin exposure, indicating that Raman can be employed to help understand cellular resistance pathways.

Finally, Nawaz et al.[48] performed a very interesting study, applying partial least squares regression analysis (PLSR) to build a predictive model on the interaction of the chemotherapeutic agent cisplatin with lung adenocarcinoma cells. In order to build the predictive model they correlated Raman measurements with the standard MTT cytotoxicity assay, and performed Raman measurements also on isolated DNA exposed to cisplatin. Using this technique, they were able to distinguish cellular responses in the Raman spectrum as a result of cisplatin interacting with the cell by chemical binding, and the subsequent 
cellular physiological response on the cell. Therefore, detailed information on the molecular level interaction of the drug was gained, and the PLSR model could be used to predict response to application of an unknown concentration of cisplatin. This information can be used to build up a database correlating spectral response to drug interactions, which could be used as a reference point for establishing 'mode of action' for new drugs spectroscopically. Keating et al.[74] went on to confirm these results with simulated data, further emphasising the power of this technique, with application to HTS for in vitro drug testing.

\section{An alternative technique - surface enhanced Raman spectroscopy (SERS)}

One of the major downfalls of Raman spectroscopy is that it is an inherently weak process, and therefore, to obtain significant signal-to-noise, longer acquisition times tend to be required. Surface enhanced Raman spectroscopy (SERS), uses the plasmonic properties of roughened metal nanoparticles, to enhance Raman signals by up to $10^{15}$.[75] SERS has become a popular technique in multiple research areas including materials and electrochemistry, as well as in biomedical research areas.[76] SERS can be used to enhance native Raman signals, by close proximity to roughened metal surfaces. However, perhaps the more promising application is to probe for specific interactions, for example by detection of specific antigens[37] or enzyme activity[18,77]. Kneipp et al.[78] demonstrated the use of SERS to enhance Raman signals in single cells, therefore decreasing the acquisition time for a single map significantly. While SERS has the downfall of being an invasive technique, this could perhaps be compensated for in HTS by its ability to rapidly increase assay speed. In this manner, SERS could potentially detect the same changes as observed in spontaneous Raman spectra in response to drug application, with much faster acquisition times and therefore assay speed.

Austin et al.[19] used a technique termed targeted plasmonically enhanced single cell imaging spectroscopy (T-PESCIS), based on enhanced SERS and Rayleigh scattering from cells treated with targeted nanoparticles. They observed changes in intensity of so called 'death-bands' in response to application of the chemotherapy drugs cisplatin and 5-fluorouracil (5-FU) to human oral squamous carcinoma cells. This allowed drug efficacy to be determined from the changing intensity of particular SERS bands, highlighting its potential for use in assays for high throughput drug screening. In this example, nanoparticles were targeted to the nuclei of cells. Nanoparticles have been targeted to various specific cell regions and characteristics for example Von-Dinh patented a technology where nanoparticles were designed to detect specific cellular components, both intracellular and extracellular.[79] This was proposed as a platform to allow high throughput drug screening, using multiple nanoprobes. Potentially multiple nanoprobes could be combined in one assay to provide multiplexed detection of an array of cellular characteristics. Multiplexing capability is one of the major advantages of SERS over fluorescence based assays.[38] The sharp spectral signals in SERS compared to the broad fluorescence signals allow for multiple probes to be used in a single assay. In terms of HTS this is greatly beneficial as it provides increased information in one assay, potentially decreasing the need for additional assays and increasing speed.

A closely related technique to SERS is surface enhanced resonance Raman scattering (SERRS) and this has been widely used in biological assays with potential HTS capability. SERRS employs a reporter 'dye' molecular that is attached to the nanoparticle and absorbs at approximately the same wavelength as the nanoparticle is resonant. Therefore even greater enhancement in signal is observed. In this case, the signal observed is that from the dye on the nanoparticle, which is turned 'on' or 'off' by the presence of, for example, a particular enzyme. By combining nanoparticles functionalised with different dye molecules and careful 
selection of combination, a multiplexed assay can be designed.[38] Nanoparticles have been functionalised with antibodies designed to detect specific antigen markers in immunoassays based on both SERS and SERRS techniques.[37,80-82] SERRS based assays have also been used to detect enzyme activity. Moore et al.[77] designed an assay to detect a variety of enzyme activities including from lipases, esterases and proteases. In these assays, a substrate consisting of a recognition site for a particular enzyme, a linker that can be cleaved by the particular enzyme and a SERRS-active dye was synthesised. In addition, silver nanoparticles were used in the assay. In the presence of the particular enzyme of interest, the substrate is cleaved, releasing the SERS-active dye, which then complexes to the surface of the nanoparticles, giving a SERS response. This assay was developed using isolated enzymes, and thus gives an example of a biochemical type assay that was proposed for use in HTS, as it allowed enzyme concentrations commonly found in vivo to be detected. SERS has also been demonstrated for detection of intracellular enzyme activity. Stevenson et al.[18] reported the first example of detection of a specific intracellular enzyme activity using SERS. In their assay, activity of the enzyme $\beta$-galactosidase was detected inside cells with subcellular resolution. The common ELISA reagent 5-bromo-4-chloro-3-indolyl $\beta$-D-galactopyranoside (X-gal) was delivered to cells along with gold nanoparticles, and a SERS response was observed when X-Gal was converted to the coloured product 5-5'-dimbromo-4,4'-dichloroindigo by $\beta$-galactosidase. The main advantages of this technique over fluorescence detection of enzyme activity were the ability to achieve subcellular resolution of enzyme activity and the multiplexing potential of the assay. It also demonstrates that, enzyme activity could be detected in a cellular as well as biochemical type assay, potentially for HTS technology.

In addition to more direct measurement of the effect of a drug on, for example, activity of a specific enzyme, a study from Jaworska et al.[83] used SERS nanosensors to measure the intracellular $\mathrm{pH}$ in endothelial cells, and how this changed in response to application of tumour necrosis factor- $\alpha$ (Figure 3). Therefore, drug compounds could be screened indirectly by measurement of physiological characteristics, in this particular example $\mathrm{pH}$, for their action. Nanosensors to measure intracellular $\mathrm{pH}$ have been reported in many studies, the most common being 4-mercaptobenzoic acid functionalised nanoparticles. [84-87] Auchinvole et al.[88] also developed nanosensors for measurement of intracellular redox potential, a key physiological characteristic, with changes in redox potential often associated with disease development. This is therefore a key characteristic that could be monitored in HTS assays to monitor response to drug treatment. An array of nanosensors with different small molecule functionalities has already been developed to measure redox potential in different intracellular redox environments.[89,90] Again, these SERS nanosensors have the benefit of multiplexing capability and have been used to measure both intracellular $\mathrm{pH}$ and redox potential simultaneously (Figure 3(e)).[91]

\section{Future perspectives: are nonlinear Raman techniques the answer?}

As mentioned, the primary downfall of Raman spectroscopy is the long acquisition times due to the inherently weak signals. Nonlinear Raman techniques, particularly stimulated Raman spectroscopy (SRS) and coherent anti-Stokes Raman spectroscopy (CARS), have emerged as incredibly promising methods for achieving high resolution label free information over much shorter acquisition times than spontaneous Raman.[92] Therefore, these nonlinear techniques could overcome the main disadvantage of spontaneous Raman spectroscopy and provide a rapid label free detection method for high throughput spectroscopic screening. CARS and SRS involve interrogating the sample with two laser beams of different frequency - a pump beam and a probe/Stokes beam.[92] Where the difference in frequency between the pump and Stokes beam match a vibrational energy transition, that vibrational transition is interrogated. 
CARS involves stimulating the coherent vibration of a chemical bond via the application of a pump and Stokes beam, which interacts with another pump photon, then leading to emission of an anti-Stokes photon. In SRS, when the pump and Stokes beam interrogate a sample with an energy difference equal to a chemical bond vibrational energy, the molecule is stimulated into the excited vibrational state. In this process energy is transferred from the pump to Stokes beam, resulting in the disappearance of the pump photon and creation of a second Stokes photon. The disadvantage of CARS, compared to SRS, is that, a non-resonant background is present in CARS measurements at the frequency of the anti-Stokes. This is not present in SRS.[92]

Significantly, CARS and SRS, as well as spontaneous Raman, have been used to study lipids and lipid metabolism in vitro. This is particularly significant as lipids are difficult to study using other techniques - they are non fluorescent and difficult to label for fluorescence studies.[92] Raman techniques give strong lipid signals and therefore open up new opportunities for studying lipids, in particular here, how they might change in response to drug treatment. CARS and SRS techniques have been widely used for the study of lipid distribution and dynamics.[93,94] Many studies have been carried out on model organisms such as Caenorhabditis elegans[94,95], showing the ability of these techniques to image lipids with high contrast. Hellerer et al.[95] report on the study of lipid storage in Caenorhabditis elegans using CARS and how genetic variations affect this. There is potential for these techniques to be extended to study how lipid storage and metabolism is altered in response to drug treatment.

In addition to potentially monitoring the effect of a drug on cellular biomolecules such as lipids, CARS and SRS have been used to image drug distribution itself, by tuning in to specific vibrational frequencies of drug molecules. This is an emerging area of research with great potential for HTS application in a label free and quick manner. Kang et al.[96] used CARS to visualize the distribution of the drug paclitaxel in polymer films in 3D. As a result of the added benefit of lack of non-resonant background in SRS compared to CARS, as well as a linear concentration dependence and lack of spectral distortion, SRS could perhaps provide the answer to high throughput spectroscopic drug screening. Tipping et al.[20] recently reviewed SRS as "an emerging tool for drug discovery". In this review, the potential for SRS as the latest development for in vitro drug screening is explored, pointing towards SRS as the future for spectroscopic HTS. In a particularly exciting study, Fu et al.[97] used hyperspectral SRS to study the uptake and distribution of two tyrosine-kinase inhibitors (TKI), imatinib and nilotinib, in murine cell line BaF3 cells. Hyperspectral SRS images a number of Raman frequencies, providing additional biochemical information regarding the drug distribution inside cells by allowing both the drug and cellular signals to be recorded. This study revealed lysosomal accumulation of the drugs, and allowed mechanisms behind the accumulation of the drugs in cells to be elucidated. Lysosomal accumulation of imatinib was decreased by addition of chloroquine, increasing cytosolic drug availability. Therefore, the information gained through hyperspectral SRS allowed detailed information on drug uptake and distribution to be gained, and methods to alter this were developed, with potential for increased efficacy. While these non-linear Raman techniques are at the early stages of development for potential spectroscopic HTS, they may be the solution to providing a label free, fast method for analysing drug uptake, distribution, metabolism, and physiological effects intracellularly. 


\section{Conclusion: high throughput spectroscopic screening - a reality?}

This review has highlighted some of the key developments in the use of vibrational spectroscopy for analysis of drug-cell interactions. HTS is a relatively recent development in biomedical research and has allowed the preclinical screening of drug candidates to be rapidly increased in speed. HTS requires detection techniques to assess the action of potential drug molecules. Typically, these assays are fluorescence based and involve monitoring a specific phenotype or specific drug-protein binding interaction. The key characteristics of such a detection mechanism are: simple, cheap, fast and efficient. Here the question is posed: could high-throughput vibrational spectroscopic screening improve drug development. In order to do so it needs to meet these key characteristics and supersede the current techniques in doing so. In this review, a number of examples have been given of how vibrational spectroscopy has been used to detect cellular response to drugs, summarised in Figure 4 in comparison to fluorescence. In particular, FTIR and Raman techniques are considered in application to cell based in vitro assays. One of the major benefits of these techniques is that they are label free - both decreasing cost due to lack of regents required, and increasing speed as this removes additional assay steps involving reagents addition and detection. Another major benefit is the wealth of information acquired in a single vibrational spectrum. Data is multivariate, and therefore can reveal detailed biochemical information on the cellular interaction of a drug candidate. While this also increases computational power required to perform multivariate data analysis, it allows both simple 'on' or 'off' response to drug to be determined by overall analysis of biochemical changes, but can also reveal more detailed and specific information potentially relating to 'mode of action' and potential side effects. This could reduce the number of assays required in the overall drug screening process. In particular, Raman spectroscopy has shown both overall drug response in addition to subcellular localisation of a drug molecule and its metabolites. While IR and Raman spectroscopy pose major advantages for HTS, there remain potential drawbacks. In IR spectroscopy, large water contributions in aqueous samples and Mie scattering pose problems, that require microfluidic devices and computer correction algorithms to get round. Raman spectroscopy provides fewer problems, with perhaps the main consideration being the cost of substrate to minimise background signal from this. While higher cost substrates are favourable, it has been shown that low cost glass is adequate with careful wavelength selection. SERS and SERRS are introduced as additional vibrational techniques with potential for HTS. These techniques have already been extensively developed for immunoassay type screening, able to detect specific antigens. Assays have also been developed for monitoring of enzyme activity both in isolated enzyme assays and inside cells. While these are invasive techniques, negating the label free advantage of IR or spontaneous Raman screening, they provide many benefits including enhanced signal compared to Raman and therefore a decrease in acquisition time. The major benefits of these assays over their fluorescent counterparts are lack of photobleaching, increased subcellular resolution and ability for multiplexing. Finally, the non-linear Raman techniques CARS and SRS, have potential to overcome the speed issues of spontaneous Raman spectroscopy, providing a fast, label free platform for spectroscopic HTS of drug candidates to elucidate on drug uptake, distribution and physiological response in vitro.

While Raman and IR may not surpass the present on/off throughput rates in terms of number of screening events in a given time, by providing a more holistic view of the biochemistry of the cell, and therefore, in the same screen allowing multiple characteristics to be probes such as toxicity, drug uptake, subcellular trafficking of drug, binding events and efficacy, the overall throughput could be increased by reducing the number of screening events required per drug candidate. By being non invasive, Raman and IR also reduce the number of steps in 
the screening process. SERS/SERRS and CARS/SRS provide a less holistic view of cellular biochemistry, instead often focusing on specific interactions or events. These techniques are faster, competing with current HTS methods. Ultimately, CARS and SRS could allow real time screening. Figure 5 summarises the potential workflow of high throughput vibrational spectroscopic screening, including reference to the various spectroscopic methods discussed and the reasons for selecting each. Brief reference is also made to sample preparation methods. As mentioned throughout the review, consideration of sample preparation is important for spectroscopic measurements. It is possible for all spectroscopic measurements to be made on both live and fixed samples although in the case of IR, fixed samples are particularly preferable due to the influence of water and the need for water correction algorithms discussed. While sample substrate can have a large affect on the quality of data acquired, as discussed, particularly in relation to IR and Raman techniques, HTS is characterised by the need for low cost. Therefore, despite background contributions, the substrate of choice would be glass. Due to the large enhancements, substrate is not important in SERS/SERRS assays and these could become the favoured method in high throughput spectroscopic screening. Therefore, the various spectroscopic methods discussed all have their merits and could be selected dependent on the screening application in question. These complimentary techniques highlight the additional advantage of vibrational spectroscopic screening by showcasing the array of 'tools' to select from dependent on the particular application in question. The spectroscopic technique employed could be selected by consideration of the points discussed in this article and summarised in the workflow in Figure 5. Perhaps, ultimately, the faster nonlinear Raman techniques of CARS and SRS, which could allow real time monitoring of drug action and localisation, hold the key to the progression of high throughput vibrational spectroscopic screening.

\section{Acknowledgements}

L. Jamieson would like to thank Caryn Hughes for her input and support.

\section{References}

[1] L.M. Almond, J. Hutchings, G. Lloyd, H. Barr, N. Shepherd, J. Day, O. Stevens, S. Sanders, M. Wadley, N. Stone, C. Kendall, Endoscopic Raman spectroscopy enables objective diagnosis of dysplasia in Barrett's esophagus, Gastrointest. Endosc. 79 (2014) 37-45. doi:10.1016/j.gie.2013.05.028.

[2] D.C. Fernandez, R. Bhargava, S.M. Hewitt, I.W. Levin, Infrared spectroscopic imaging for histopathologic recognition., Nat. Biotechnol. 23 (2005) 469-474. doi:10.1038/nbt1080.

[3] A. Khoshmanesh, M.W.A. Dixon, S. Kenny, L. Tilley, D. McNaughton, B.R. Wood, Detection and Quantification of Early-Stage Malaria Parasites in Laboratory Infected Erythrocytes by Attenuated Total Reflectance Infrared Spectroscopy and Multivariate Analysis., Anal. Chem. 86 (2014) 4379-4386. doi:10.1021/ac500199x.

[4] L. Lovergne, G. Clemens, V. Untereiner, R.A. Lukaszweski, G.D. Sockalingum, M.J. Baker, Investigating optimum sample preparation for infrared spectroscopic serum diagnostics, Anal. Methods. 7 (2015) 7140-7149. doi:10.1039/C5AY00502G.

[5] F.M. Lyng, D. Traynor, I.R.M. Ramos, F. Bonnier, H.J. Byrne, Raman spectroscopy for screening and diagnosis of cervical cancer, Anal. Bioanal. Chem. 407 (2015) 8279-8289. doi:10.1007/s00216-015-8946-1.

[6] H.J. Byrne, M. Baranska, G.J. Puppels, N. Stone, B. Wood, K.M. Gough, P. Lasch, P. Heraud, J. Sulé-Suso, Spectropathology for the next generation: Quo vaids?, Analyst. 140 (2015) 2066-2073. doi:10.1039/c4an02036g.

[7] The International Society for Clinical Spectroscopy, http://clirspec.org (accessed April 8, 2016). 
[8] Raman4Clinics, https://www.raman4clinics.eu (accessed May 24, 2016).

[9] Laser detects brain tumour cells during surgery, http://www.bbc.co.uk/news/health34041863 (accessed September 6, 2015).

[10] M. Jermyn, K. Mok, J. Mercier, J. Desroches, J. Pichette, K. Saint-Arnaud, L. Bernstein, M.-C. Guiot, K. Petrecca, F. Leblond, Intraoperative brain cancer detection with Raman spectroscopy in humans, Sci. Transl. Med. 7 (2015) 274ra19-274ra19. doi:10.1126/scitranslmed.aaa2384.

[11] Infrared light puts malaria to the test, http://monash.edu/news/show/infrared-lightputs-malaria-to-the-test (accessed September 6, 2015).

[12] Legislation for the protection of animals used for scientific purposes, http://ec.europa.eu/environment/chemicals/lab_animals/legislation_en.htm (accessed May 15, 2016).

[13] Public Law 106-387 106th Congress An Act, (2001).

[14] A.A. Hajare, S.S. Salunkhe, S.S. Mali, S.S. Gorde, S.J. Nadaf, S.A. Pishawikar, Review on: High-throughput screening is an approach to drug discovery, Am. J. PharmTech Res. 4 (2013) 112-129.

[15] C. Hughes, G. Clemens, M.J. Baker, Preclinical screening of anticancer drugs using infrared (IR) microspectroscopy, Trends Biotechnol. 33 (2015) 429-430. doi:10.1016/j.tibtech.2015.03.010.

[16] H. Nawaz, F. Bonnier, P. Knief, O. Howe, F.M. Lyng, A.D. Meade, H.J. Byrne, Evaluation of the potential of Raman microspectroscopy for prediction of chemotherapeutic response to cisplatin in lung adenocarcinoma., Analyst. 135 (2010) 3070-3076. doi:10.1039/c0an00541j.

[17] A. Derenne, R. Gasper, E. Goormaghtigh, The FTIR spectrum of prostate cancer cells allows the classification of anticancer drugs according to their mode of action., Analyst. 136 (2011) 1134-1141. doi:10.1039/c0an00872a.

[18] R. Stevenson, S. McAughtrie, L. Senior, R.J. Stokes, H. McGachy, L. Tetley, P. Nativo, J.M. Brewer, J. Alexander, K. Faulds, D. Graham, Analysis of intracellular enzyme activity by surface enhanced Raman scattering., Analyst. 138 (2013) 6331-6336. doi:10.1039/c3an00729d.

[19] L.A. Austin, B. Kang, M.A. El-Sayed, A New Nanotechnology Technique for Determining Drug E ffi cacy Using Targeted Plasmonically Enhanced Single Cell Imaging Spectroscopy, J. Am. Chem. Soc. 135 (2013) 4688-4691. doi: 10.1021/ja4011145.

[20] W.J. Tipping, M. Lee, A. Serrels, V.G. Brunton, A.N. Hulme, Stimulated Raman scattering microscopy: an emerging tool for drug discovery, Chem. Soc. Rev. 45 (2016) 2075-2089. doi:10.1039/C5CS00693G.

[21] P. Szymański, M. Markowicz, E. Mikiciuk-Olasik, Adaptation of high-throughput screening in drug discovery-toxicological screening tests, Int. J. Mol. Sci. 13 (2012) 427452. doi:10.3390/ijms13010427.

[22] D.L. Boger, B.E. Fink, M.P. Hedrick, Total synthesis of distamycin A and 2640 analogues: A solution-phase combinatorial approach to the discovery of new, bioactive DNA binding agents and development of a rapid, high-throughput screen for determining relative DNA binding affinity or DNA binding selectivity, J. Am. Chem. Soc. 122 (2000) 6382-6394. doi:10.1021/ja994192d.

[23] I. Torjesen, Drug development: the journey of a medicine from lab to shelf, Pharm. J. (2015). http://www.pharmaceutical-journal.com/publications/tomorrowspharmacist/drug-development-the-journey-of-a-medicine-from-lab-toshelf/20068196.article (accessed April 8, 2016).

[24] D. Barata, C. van Blitterswijk, P. Habibovic, High-throughput screening approaches and combinatorial development of biomaterials using microfluidics, Acta Biomater. 34 (2015) 1-20. doi:10.1016/j.actbio.2015.09.009. 
[25] N. Boute, R. Jockers, T. Issad, The use of resonance energy transfer in high-throughput screening: BRET versus FRET, Trends Pharmacol. Sci. 23 (2002) 351-354. doi:10.1016/S0165-6147(02)02062-X.

[26] D.S. Smith, S.A. Eremin, Fluorescence polarization immunoassays and related methods for simple, high-throughput screening of small molecules, Anal. Bioanal. Chem. 391 (2008) 1499-1507. doi:10.1007/s00216-008-1897-z.

[27] Y. Jia, C.M. Quinn, A.I. Gagnon, R. Talanian, Homogeneous time-resolved fluorescence and its applications for kinase assays in drug discovery, Anal. Biochem. 356 (2006) 273-281. doi:10.1016/j.ab.2006.05.006.

[28] M. Auer, K.J. Moore, F.J. Meyer-Almes, R. Guenther, A.J. Pope, K.A. Stoeckli, Fluorescence correlation spectroscopy: Lead discovery by miniaturized HTS, Drug Discov. Today. 3 (1998) 457-465. doi:10.1016/S1359-6446(98)01240-9.

[29] J. Inglese, R.L. Johnson, A. Simeonov, M. Xia, W. Zheng, C.P. Austin, D.S. Auld, Highthroughput screening assays for the identification of chemical probes., Nat. Chem. Biol. 3 (2007) 466-479. doi:10.1038/nchembio.2007.17.

[30] A.M. Maffia III, I. Kariv, K.R. Oldenburg, Miniaturization of a Mammalian Cell-Based Assay: Luciferase Reporter Gene Readout in a 3 Microliter 1536-Well Plate, J. Biomol. Screen. 4 (1999) 137-142. doi:10.1177/108705719900400307.

[31] L.A. Collins, M.N. Torrero, S.G. Franzblau, Green fluorescent protein reporter microplate assay for high-throughput screening of compounds against Mycobacterium tuberculosis, Antimicrob. Agents Chemother. 42 (1998) 344-347.

[32] M. Berg, K. Undisz, R. Thiericke, T. Moore, C. Posten, Miniaturization of an enzyme assay ( $\beta$-galactosidase) in the 384- and 1536-well plate format, J. Assoc. Lab. Autom. 4 (1999) 64-67. doi:10.1016/S1535-5535(04)00042-5.

[33] Y-M. Go, D.P. Jones, Redox compartmentalization in eukaryotic cells, Biochim. Biophys. Acta., 1780 (2009) 1273-1290. doi:10.1016/j.bbagen.2008.01.011.

[34] R. Hamid, Y. Rotshteyn, L. Rabadi, R. Parikh, P. Bullock, Comparison of alamar blue and MTT assays for high through-put screening, Toxicol. Vitr. 18 (2004) 703-710. doi:10.1016/j.tiv.2004.03.012.

[35] V. Vichai, K. Kirtikara, Sulforhodamine B colorimetric assay for cytotoxicity screening., Nat. Protoc. 1 (2006) 1112-1116. doi:10.1038/nprot.2006.179.

[36] High Throughput Raman Spectrometer, http://www.is-instruments.com/highthroughput-raman-spectrometer.html (accessed April 25, 2016).

[37] R. Stevenson, A. Ingram, H. Leung, D.C. McMillan, D. Graham, Quantitative SERRS immunoassay for the detection of human PSA., Analyst. 134 (2009) 842-844. doi:10.1039/b902174d.

[38] S. Laing, K. Gracie, K. Faulds, Multiplex in vitro detection using SERS, Chem. Soc. Rev. 45 (2016) 1901-1918. doi:10.1039/C5CS00644A.

[39] H.J. Byrne, P. Knief, M.E. Keating, F. Bonnier, Spectral pre and post processing for infrared and Raman spectroscopy of biological tissues and cells, Chem. Soc. Rev. 45 (2016) 1865-1878. doi:10.1039/C5CS00440C.

[40] P. Bassan, A. Kohler, H. Martens, J. Lee, H.J. Byrne, P. Dumas, E. Gazi, M. Brown, N. Clarke, P. Gardner, Resonant Mie scattering (RMieS) correction of infrared spectra from highly scattering biological samples., Analyst. 135 (2010) 268-277. doi:10.1039/b921056c.

[41] S.W. Bruun, A. Kohler, I. Adt, G.D. Sockalingum, M. Manfait, H. Martens, Correcting attenuated total reflection-fourier transform infrared spectra for water vapor and carbon dioxide, Appl. Spectrosc. 60 (2006) 1029-1039. doi:10.1366/000370206778397371.

[42] L.T. Kerr, H. Byrne, B.M. Hennelly, Optimal choice of sample substrate and laser wavelength for Raman spectroscopic analysis of biological specimen, Anal. Methods. 7 (2015) 5041-5052. doi:10.1039/C5AY00327J. 
[43] K.H. Liland, A. Kohler, N.K. Afseth, Model-based pre-processing in Raman spectroscopy of biological samples, J. Raman Spectrosc. (2016). doi:10.1002/jrs.4886.

[44] M.J. Baker, C.S. Hughes, K.A. Hollywood, Biophotonics: Vibrational Spectroscopic Diagnostics, Morgan \& Claypool Publishers, 2016. doi:10.1088/978-1-6817-4071-3.

[45] B. Bird, M.J. Baker, Quantum Cascade Lasers in Biomedical Infrared Imaging, Trends Biotechnol. 33 (2015) 557-558. doi:10.1016/j.tibtech.2015.07.003.

[46] S.F. El-Mashtoly, D. Petersen, H.K. Yosef, A. Mosig, A. Reinacher-Schick, C. Kötting, K. Gerwert, Label-free imaging of drug distribution and metabolism in colon cancer cells by Raman microscopy., Analyst. 139 (2014) 1155-1161. doi:10.1039/c3an01993d.

[47] Z. Farhane, F. Bonnier, A. Casey, H.J. Byrne, Raman micro spectroscopy for in vitro drug screening: subcellular localisation and interactions of doxorubicin, Analyst. 140 (2015) 4212-4223. doi:10.1039/c5an00256g.

[48] H. Nawaz, F. Bonnier, P. Knief, O. Howe, F. M. Lyng, A. D. Meade, H. J. Byrne, Evaluation of the potential of Raman microspectroscopy for prediction of chemotherapeutic response to cisplatin in lung adenocarcinoma., Analyst. 135 (2010) 3070-6. doi:10.1039/c0an00541j.

[49] M. Miljković, T. Chernenko, M.J. Romeo, B. Bird, C. Matthäus, M. Diem, Label-free imaging of human cells: algorithms for image reconstruction of Raman hyperspectral datasets., Analyst. 135 (2010) 2002-13. doi:10.1039/c0an00042f.

[50] M.E. Keating, F. Bonnier, H.J. Byrne, Spectral cross-correlation as a supervised approach for the analysis of complex Raman datasets: the case of nanoparticles in biological cells, Analyst. (2012) 5792-5802. doi:10.1039/c2an36169h.

[51] P. Lasch, M. Diem, W. Hänsch, D. Naumann, Artificial neural networks as supervised techniques for FT-IR microspectrocopic imaging. J. Chemometrics 20 (2006) 209-220. doi:10.1002/cem993.

[52] A. Gaigneaux, J.M. Ruysschaert, E. Goormaghtigh, Infrared spectroscopy as a tool for discrimination between sensitive and multiresistant K562 cells, Eur. J. Biochem. 269 (2002) 1968-1973. doi:10.1046/j.1432-1327.2002.02841.x.

[53] L.M. Miller, P. Dumas, Chemical imaging of biological tissue with synchrotron infrared light, Biochim. Biophys. Acta - Biomembr. 1758 (2006) 846-857. doi:10.1016/j.bbamem.2006.04.010.

[54] A. Derenne, V. Van Hemelryck, D. Lamoral-Theys, R. Kiss, E. Goormaghtigh, FTIR spectroscopy: A new valuable tool to classify the effects of polyphenolic compounds on cancer cells, Biochim. Biophys. Acta - Mol. Basis Dis. 1832 (2013) 46-56. doi:10.1016/j.bbadis.2012.10.010.

[55] R. Gasper, T. Mijatovic, A. Bénard, A. Derenne, R. Kiss, E. Goormaghtigh, FTIR spectral signature of the effect of cardiotonic steroids with antitumoral properties on a prostate cancer cell line, Biochim. Biophys. Acta - Mol. Basis Dis. 1802 (2010) 10871094. doi:10.1016/j.bbadis.2010.07.012.

[56] F. Nie, X.-L. Yu, X.-G. Wang, Y.-F. Tang, L.-L. Wang, L. Ma, Down-regulation of CacyBP is associated with poor prognosis and the effects on COX-2 expression in breast cancer., Int. J. Oncol. 37 (2010) 1261-1269. doi:10.3892/ijo.

[57] G. Berger, H. Leclercqz, A. Derenne, M. Gelbcke, E. Goormaghtigh, J. Nève, V. Mathieu, F. Dufrasne, Synthesis and in vitro characterization of platinum(II) anticancer coordinates using FTIR spectroscopy and NCI COMPARE: A fast method for new compound discovery, Bioorganic Med. Chem. 22 (2014) 3527-3536. doi:10.1016/j.bmc.2014.04.017.

[58] M.J. Baker, J. Trevisan, P. Bassan, R. Bhargava, H.J. Butler, K.M. Dorling, P.R. Fielden, S.W. Fogarty, N.J. Fullwood, K.A. Heys, C. Hughes, P. Lasch, P.L. Martin-Hirsch, B. Obinaju, G.D. Sockalingum, J. Sulé-Suso, R.J. Strong, M.J. Walsh, B.R. Wood, P. Gardner, F.L. Martin, Using Fourier transform IR spectroscopy to analyze biological 
[59] Perkin Elmer, Technical Note, The Effects of Varying Force and Contact on ATR Spectra, Tech. Note. (2012) 4-6.

[60] E. Levenson, P. Lerch, M.C. Martin, Spatial resolution limits for synchrotron-based spectromicroscopy in the mid- and near-infrared, J. Synchrotron Radiat. 15 (2008) 323328. doi:10.1107/S0909049508004524.

[61] C. Hughes, A. Henderson, M. Kansiz, K. Dorling, M. Jimenez-Hernandez, M.D. Brown, N.W. Clarke, P. Gardner, Enhanced FTIR benchtop imaging of single biological cells, Analyst. (2015) 2080-2085. doi:10.1039/C4AN02053G.

[62] F. Draux, P. Jeannesson, C. Gobinet, J. Sulé-Suso, J. Pijanka, C. Sandt, P. Dumas, M. Manfait, G.D. Sockalingum, IR spectroscopy reveals effect of non-cytotoxic doses of anti-tumour drug on cancer cells, Anal. Bioanal. Chem. 395 (2009) 2293-2301. doi:10.1007/s00216-009-3140-y.

[63] C. Hughes, M.D. Brown, F.J. Ball, G. Monjardez, N.W. Clarke, K.R. Flower, P. Gardner, Highlighting a need to distinguish cell cycle signatures from cellular responses to chemotherapeutics in SR-FTIR spectroscopy., Analyst. 137 (2012) 57365742. doi:10.1039/c2an35633c.

[64] A. Derenne, A. Mignolet, E. Goormaghtigh, FTIR spectral signature of anticancer drug effects on PC-3 cancer cells: is there any influence of the cell cycle?, Analyst. 138 (2013) 3998-4005. doi:10.1039/c3an00225j.

[65] H-Y.N. Holman, M..C. Martin, E.A. Blakely, K. Bjornstad, W.R. McKinney, IR Spectroscopic Characteristics of Cell Cycle and Cell Death Probed by Synchrotron Radiation Based Fourier Transform IR Spectromicroscopy, Biopolymers. 57 (2000) 329335. doi: 10.1002/1097-0282.

[66] M. Jimenez-Hernandez, M.D. Brown, C. Hughes, N.W. Clarke, P. Gardner, Characterising cytotoxic agent action as a function of the cell cycle using fourier transform infrared microspectroscopy., Analyst. 140 (2015) 4453-4464.

doi:10.1039/c5an00671f.

[67] S. Gaudenzi, D. Pozzi, P. Toro, I. Silvestri, S. Morrone, A.C. Castellano, Cell apoptosis specific marker found by Fourier Transform Infrared Spectroscopy, Spectroscopy. 18 (2004) 415-422. doi:10.1155/2004/483591.

[68] K-Z. Lui, J. Li, S.M. Kelsey, H.H. Mantsch, Quantitative determination of apoptosis on leukaemia cells by infrared spectroscopy., Apoptosis. 6 (2001) 267-276. doi:10.1023/A:1011383408381.

[69] A. V. Rutter, M.R. Siddique, J. Filik, C. Sandt, P. Dumas, G. Cinque, G.D. Sockalingum, Y. Yang, J. Sulé-Suso, Study of gemcitabine-sensitive/resistant cancer cells by cell cloning and synchrotron FTIR microspectroscopy, Cytom. Part A. 85 (2014) 688-697. doi:10.1002/cyto.a.22488.

[70] F. Bonnier, S.M. Ali, P. Knief, H. Lambkin, K. Flynn, V. McDonagh, C. Healy, T.C. Lee, F.M. Lyng, H.J. Byrne, Analysis of human skin tissue by Raman microspectroscopy: Dealing with the background, Vib. Spectrosc. 61 (2012) 124-132. doi:10.1016/j.vibspec.2012.03.009.

[71] R. Buckmaster, F. Asphahani, M. Thein, J. Xu, M. Zhang, Detection of drug-induced cellular changes using confocal Raman spectroscopy on patterned single-cell biosensors., Analyst. 134 (2009) 1440-1446. doi:10.1039/b900420c.

[72] Z. Farhane, F. Bonnier, A. Casey, H. Byrne, Raman micro spectroscopy for in vitro drug screening: subcellular localisation and interactions of Doxorubicin, Analyst. (2015) 4212-4223. doi:10.1039/C5AN00256G.

[73] Z. Farhane, F. Bonnier, B. Marcus Alexander, Maher Jane, C. Alan, H.J. Byrne, Differentiating responses of lung cancer cell lines to Doxorubicin exposure: in vitro Raman micro spectroscopy, oxidative stress and bcl-2 protein expression, J. 
Biophotonics. (2016) 1-15. doi:10.1002/jbio.201600019.

[74] M.E. Keating, H. Nawaz, F. Bonnier, H.J. Byrne, Multivariate statistical methodologies applied in biomedical Raman spectroscopy: assessing the validity of partial least squares regression using simulated model datasets, Analyst. 140 (2015) 2482-2492. doi:10.1039/c4an02167c.

[75] E.C. Le Ru, P.G. Etchegoin, Principles of Surface-Enhanced Raman Spectroscopy, Chapter 4 SERS enhancement factors and related topics, Elsevier (2009). doi:10.1016/B978-0-444-52779-0.00009-X.

[76] Z.Q. Tian, Surface-enhanced Raman spectroscopy: Advancements and applications, J. Raman Spectrosc. 36 (2005) 466-470. doi:10.1002/jrs.1378.

[77] B.D. Moore, L. Stevenson, A. Watt, S. Flitsch, N.J. Turner, C. Cassidy, D. Graham, Rapid and ultra-sensitive determination of enzyme activities using surface-enhanced resonance Raman scattering., Nat. Biotechnol. 22 (2004) 1133-1138. doi:10.1038/nbt1003.

[78] K. Kneipp, A.S. Haka, H. Kneipp, K. Badizadegan, N. Yoshizawa, C. Boone, K.E. Shafer-Peltier, J.T. Motz, R.R. Dasari, M.S. Feld, Surface-enhanced raman spectroscopy in single living cells using gold nanoparticles, Appl. Spectrosc. 56 (2002) 150-154. doi:10.1366/0003702021954557.

[79] T. Vo-Dinh, Nanoprobe for surface-enhanced Raman spectroscopy in medical diagnostic and drug screening, 2001, US 6,219,137 B1, filed 3 ${ }^{\text {rd }}$ Dec 1998, issued 17th April 2001.

[80] D.S. Grubisha, R.J. Lipert, H.Y. Park, J. Driskell, M.D. Porter, Femtomolar Detection of Prostate-Specific Antigen: An Immunoassay Based on Surface-Enhanced Raman Scattering and Immunogold Labels, Anal. Chem. 75 (2003) 5936-5943. doi:10.1021/ac034356f.

[81] J. Ni, R.J. Lipert, G.B. Dawson, M.D. Porter, Immunoassay Readout Method Using Extrinsic Raman Labels Adsorbed on Immunogokld Collids, Anal. Chem. 71 (1999) 4903-4908. doi:10.1021/ac990616a.

[82] X.X. Han, B. Zhao, Y. Ozaki, Surface-enhanced Raman scattering for protein detection, Anal. Bioanal. Chem. 394 (2009) 1719-1727. doi:10.1007/s00216-009-2702-3.

[83] A. Jaworska, L.E. Jamieson, K. Malek, C.J. Campbell, J. Choo, S. Chlopicki, M. Baranska, SERS-based monitoring of the intracellular $\mathrm{pH}$ in endothelial cells: the influence of the extracellular environment and tumour necrosis factor- $\alpha$, Analyst. 140 (2015) 2321-2329. doi:10.1039/c4an01988a.

[84] S.W. Bishnoi, C.J. Rozell, C.S. Levin, M.K. Gheith, B.R. Johnson, D.H. Johnson, N.J. Halas, All-optical nanoscale pH meter., Nano Lett. 6 (2006) 1687-1692. doi:10.1021/n1060865w.

[85] M.A. Ochsenkuehn, P.R.T. Jess, H. Stoquert, K. Dholakia, C.J. Campbell, Nanoshells for Surface-Enhanced Raman, ACS Nano. 3 (2009) 3613-3621. doi:10.1021/nn900681c.

[86] J. Kneipp, H. Kneipp, B. Wittig, K. Kneipp, Following the Dynamics of pH in Endosomes of Live Cells with SERS Nanosensors, J. Phys. Chem. C. 114 (2010) 74217426. doi:10.1021/jp910034z.

[87] J. Kneipp, H. Kneipp, B. Wittig, K. Kneipp, One- and Two-Photon Excited Optical pH Probing for Cells Using Surface-Enhanced Raman and Hyper-Raman Nanosensors, Nano Lett. 7 (2007) 2819-2823. doi:10.1021/n1071418z.

[88] C.A.R. Auchinvole, P. Richardson, C. McGuinnes, V. Mallikarjun, K. Donaldson, H. McNab, C.J. Campbell, Monitoring intracellular redox potential changes using SERS nanosensors., ACS Nano. 6 (2012) 888-896. doi:10.1021/nn204397q.

[89] J. Jiang, C. Auchinvole, K. Fisher, C.J. Campbell, Quantitative measurement of redox potential in hypoxic cells using SERS nanosensors., Nanoscale. 6 (2014) 12104-12110. doi:10.1039/c4nr01263a. 
[90] P.I.T. Thomson, V.L. Camus, Y. Hu, C.J. Campbell, Series of Quinone-Containing Nanosensors for Biologically Relevant Redox Potential Determination by SurfaceEnhanced Raman Spectroscopy, Anal. Chem. 87 (2015) 4719-4725. doi:10.1021/ac504795s.

[91] L.E. Jamieson, A. Jaworska, J. Jiang, M. Baranska, D.J. Harrison, C.J. Campbell, Simultaneous intracellular redox potential and $\mathrm{pH}$ measurements in live cells using SERS nanosensors, Analyst. 140 (2015) 2330-2335. doi:10.1039/c4an02365j.

[92] A. Folick, W. Min, M.C. Wang, Label-free imaging of lipid dynamics using Coherent Anti-stokes Raman Scattering (CARS) and Stimulated Raman Scattering (SRS) microscopy, Curr. Opin. Genet. Dev. 21 (2011) 585-590. doi:10.1016/j.gde.2011.09.003.

[93] J.P. Pezacki, J.A. Blake, D.C. Danielson, D.C. Kennedy, R.K. Lyn, R. Singaravelu, Chemical contrast for imaging living systems: molecular vibrations drive CARS microscopy, Nat. Chem. Biol. 7 (2011) 137-145. doi:10.1038/nchembio.525.

[94] C.W. Freudiger, W. Min, G.R. Holtom, B. Xu, M. Dantus, X. Sunney Xie, X.S. Xie, Highly specific label-free molecular imaging with spectrally tailored excitationstimulated Raman scattering (STE-SRS) microscopy, Nat. Photonics. 5 (2011) 103-109. doi:10.1038/nphoton.2010.294.

[95] T. Hellerer, C. Axäng, C. Brackmann, P. Hillertz, M. Pilon, A. Enejder, Monitoring of lipid storage in Caenorhabditis elegans using coherent anti-Stokes Raman scattering (CARS) microscopy., Proc. Natl. Acad. Sci. U. S. A. 104 (2007) 14658-14663. doi:10.1073/pnas.0703594104.

[96] E. Kang, H. Wang, I.K. Kwon, J. Robinson, K. Park, J. Cheng, In Situ Visualization of Paclitaxel Distribution and Release by Coherent Anti-Stokes Raman Scattering Microscopy In Situ Visualization of Paclitaxel Distribution and Release by Coherent Anti-Stokes Raman Scattering Microscopy, Anal. Chem. 78 (2006) 8036-8043. doi:10.1021/ac061218s.

[97] D. Fu, J. Zhou, W.S. Zhu, P.W. Manley, Y.K. Wang, T. Hood, A. Wylie, X.S. Xie, Imaging the intracellular distribution of tyrosine kinase inhibitors in living cells with quantitative hyperspectral stimulated Raman scattering., Nat. Chem. 6 (2014) 614-622. doi:10.1038/nchem.1961. 
Figure 1 FTIR spectroscopy for monitoring drug-cell interaction: Comparison between fluorescence for distinguishing cell cycle stage in Caki-2 cells and response to cytotoxic drugs 5-fluorouracil (5FU) and Paclitaxel. (a) Fluorescence on Caki-2 cells stained with DAPI (blue/cyan), cdt1-FITC (green), germinin-TRITC (yellow) and EdU-HcRed (red), with cell cycle stage labeled. (b) Caki-2 cells stained with activated caspase-3 (green) and DAPI (blue) and untreated (top), treated with 5FU (middle) and treated with Paclitaxel (bottom). (c) A selected region of the second derivative spectra of Caki-2 cells control (red), treated with 5FU (green) and treated with Paclitaxel (blue) at different cell cycle stages labeled. (d) PLS regression score plot for Caki-2 cells in $\mathrm{G}_{1}$ phase treated with $5 \mathrm{FU}$ (pink) and Paclitaxel (black) (left) and centroids of the populations from 5FU, Paclitaxel and control projection (blue). (4) PLS regression score plot for Caki-2 cells in S phase treated with 5FU (pink) and Paclitaxel (black) (left) and centroids of the populations from 5FU, Paclitaxel and control projection (green). (d) PLS regression score plot for Caki-2 cells in $\mathrm{G}_{2} \mathrm{M}$ phase treated with $5 \mathrm{FU}$ (pink) and Paclitaxel (black) (left) and centroids of the populations from 5FU, Paclitaxel and control projection (red). (g) FTIR spectroscopy has been used to distinguish cell cycle stage and response to cytotoxic drug action discriminated between each stage. (Adapted from JimenezHernandez et al.[66] with permission from the Royal Society of Chemistry.)

(a)

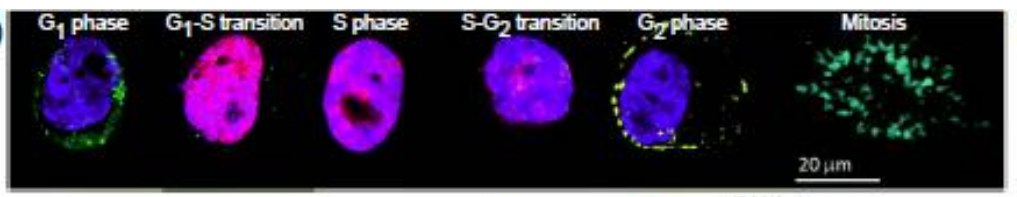

(d)

(c)



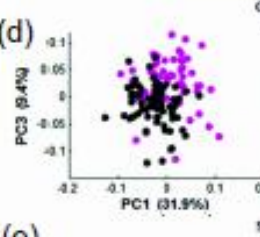

(e),



(x,

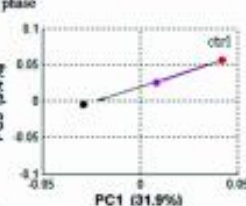

splase
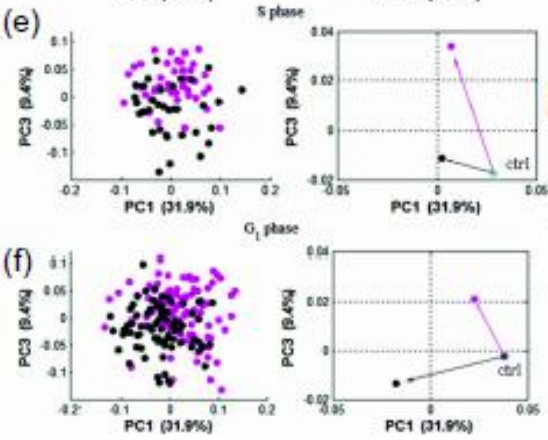

PC1 (31.96) (b)



(g)

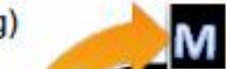


Figure 2 Raman spectroscopy for monitoring drug-cell interaction: Comparing fluorescence for imaging subcellular localization of cytotoxic drug doxorubicin and Raman spectroscopy to distinguish cellular response with subcellular distinction. (a) Confocal fluorescence image of A549 lung cancer cells control. (b) Confocal fluorescence image of A549 lung cancer cells treated with doxorubicin. (c) Mean Raman spectra of A549 cell nucleolus (green $=$ control, black $=$ doxorubicin treated). (d) Mean Raman spectra of A549 cell nucleus (red $=$ control, magenta $=$ doxorubicin treated). (e) Mean Raman spectra of A549 cell cytoplasm (blue $=$ control, cyan $=$ doxorubicin treated). $(\mathrm{f})$ PCA plot of Raman spectra from A549 cell nuclei (red $=$ untreated, yellow $=$ doxorubicin treated), nucleolus ( $g r e e n=$ untreated, black $=$ doxorubicin treated), and cytoplasm (blue $=$ untreated, pink $=$ doxorubicin treated). (g) Raman spectroscopy has been used to distinguish subcellular organelles and discriminated response to cytotoxic drug action. (Adapted from Farhane et al.[72] with permission from the Royal Society of Chemistry. Note non-IUPAC format of $x$-axis used in order to maintain consistency with how material originally appeared in publication.)

(a)

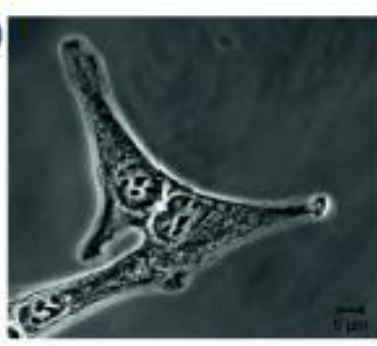

(d)



(f)



(b)



(e)

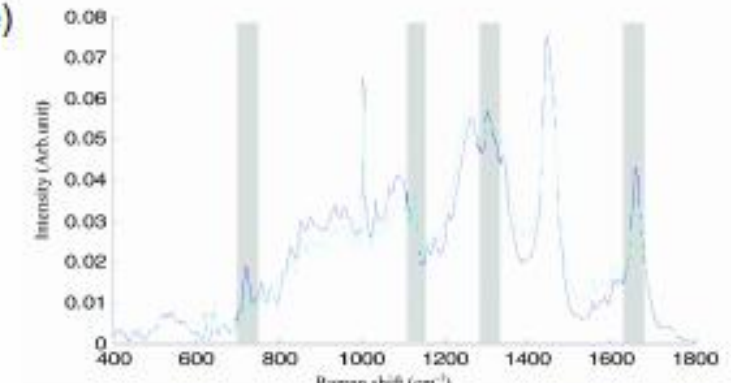

(g)

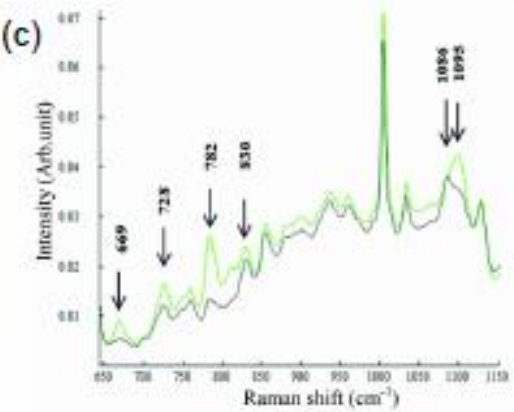




Figure 3 SERS for measuring intracellular redox potential and $\mathrm{pH}$ and changes in response to drug treatment (a) SERS spectrum of mercaptobenzoic acid functionalised gold nanoparticles (MBA-NP) at $\mathrm{pH} 1$ (black) and $\mathrm{pH} 12$ (red) showing the changing intensities of the pH sensitive signals at ca. $1400 \mathrm{~cm}^{-1}$ and ca. $1700 \mathrm{~cm}^{-1}$. (b) EA.hy926 cells incubated with MBA-NPs and mapped on Raman spectrometer to locate SERS signals from MBA-NPs inside cells, with false colour map created using cluster analysis to assign $\mathrm{pH}$ values as indicated in key. (c) and (d) Ea.hy926 cells incubated with MBA-NPs and treated with tumour necrosis factor- $\alpha$, followed by mapping on Raman spectrometer to locate SERS signals from MBA-NPs inside cells, with false colour map created using cluster analysis to assign $\mathrm{pH}$ values as indicated in key. (e) EA.hy926 cells incubated with MBA-NPs (for pH measurement) and AQ-NPs (for redox potential measurement), and mapped on Raman spectrometer to locate SERS signals from MBA-NPs and AQ-NPs inside cells, with false colour map created using cluster analysis to assign $\mathrm{pH}$ and redox potential values as indicated in the key. (f) SERS has been used to measure intracellular $\mathrm{pH}$ and its response to drug treatement. (Adapted from Jaworska et al.[83] and Jamieson et al.[91] with permission from the Royal Society of Chemistry. Note non-IUPAC format of $x$-axis used in order to maintain consistency with how material originally appeared in publication.)

(a)

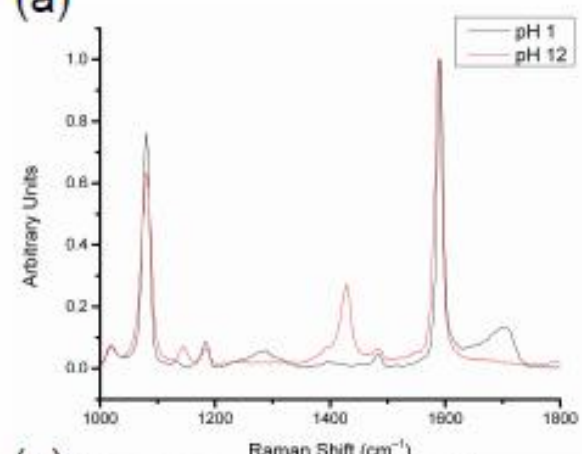

(c)

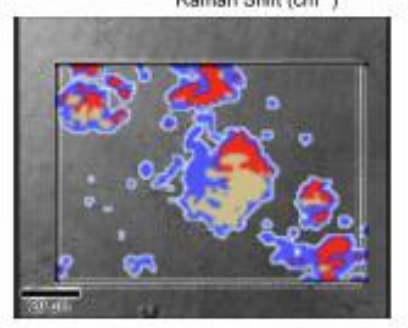

(e)



$\mathrm{pH}=5.46$ (b)



$\mathrm{pH}=6.51$

$\mathrm{pH}=6.83$

$\mathrm{pH}=7.52$

$\mathrm{pH}=7.65$

$\mathrm{pH}=\mathbf{8 . 8 0}$

$\mathrm{pH}=9.81$

(d)

$\mathrm{pH}=6.35$

$\mathrm{pH}=7.11$

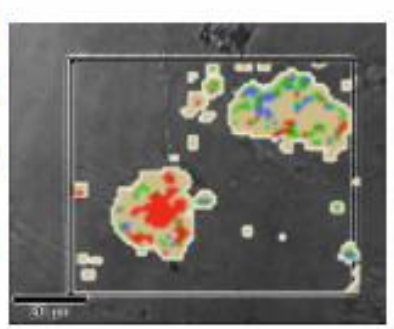

$\mathrm{pH}=6.10$

$\mathrm{pH}=6.36$

$\mathrm{pH}=6.90$

$\mathrm{pH}=7.16$

(f)

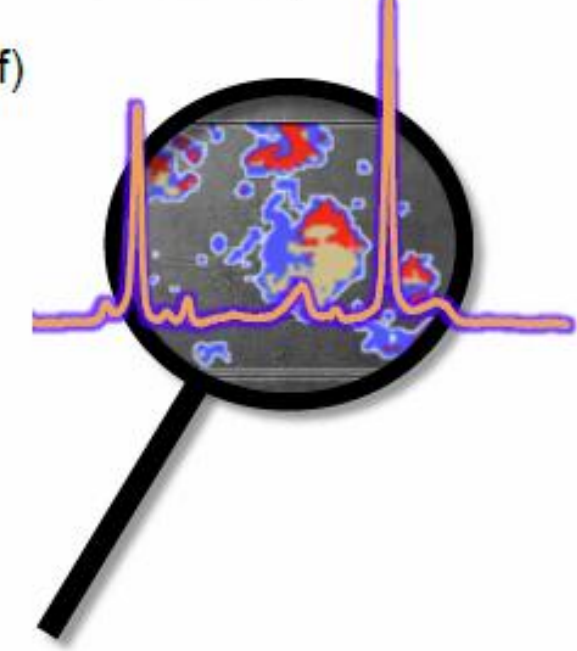


Figure 4 High throughput vibrational spectroscopic screening Vibrational spectroscopic techniques have significant potential to shape the field of HTS and provide great advantages over current fluorescent based assay techniques. Some of the advantages and disadvantages of fluorescence, FTIR spectroscopy, Raman spectroscopy, SERS and CARS/SRS as detection methods for HTS are highlighted.



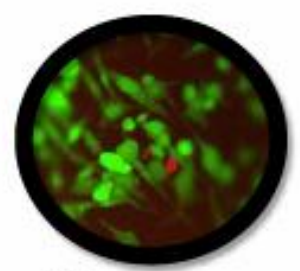

Fluorescence

$$
\begin{gathered}
\text { 'ON'rOFF' } \\
\text { Univariate } \\
\text { Photobleaching } \\
\text { Invasive }
\end{gathered}
$$

Single interaction probed



FTIR Spectroscopy Multivariate Label free 'Mode of action' Complex data processing Water and Mie scattering corrections required

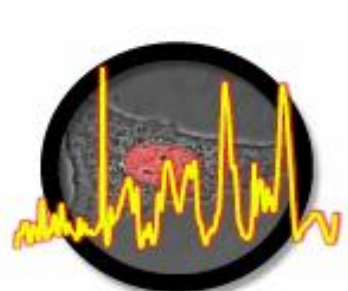

Raman Spectroscopy

$$
\begin{gathered}
\text { Multivariate } \\
\text { Label free }
\end{gathered}
$$$$
\text { Mode of action' }
$$

Subcellular information

Complex data processing

Longer acquisition time

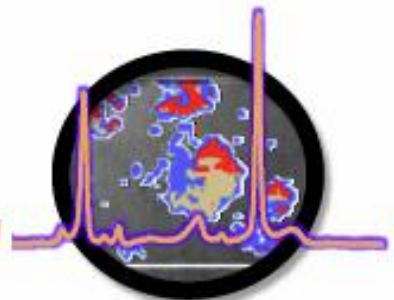

SERS

Invasive

No photobleaching

Ability to multiplex

Fast acqusition

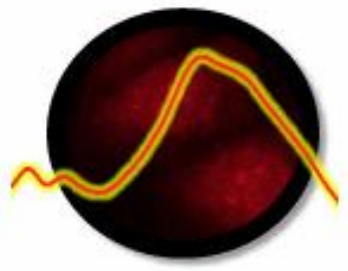

CARS/SRS

Label free Fast

'Mode of action Potentially multivariate 
Figure 5 High throughput vibrational spectroscopic screening workflow A step by step guide to high throughput vibrational spectroscopic screening from sample preparation to screening type and the factors to consider when selecting the vibrational spectroscopic method selected for screening, including pre- and post- processing data analysis steps.

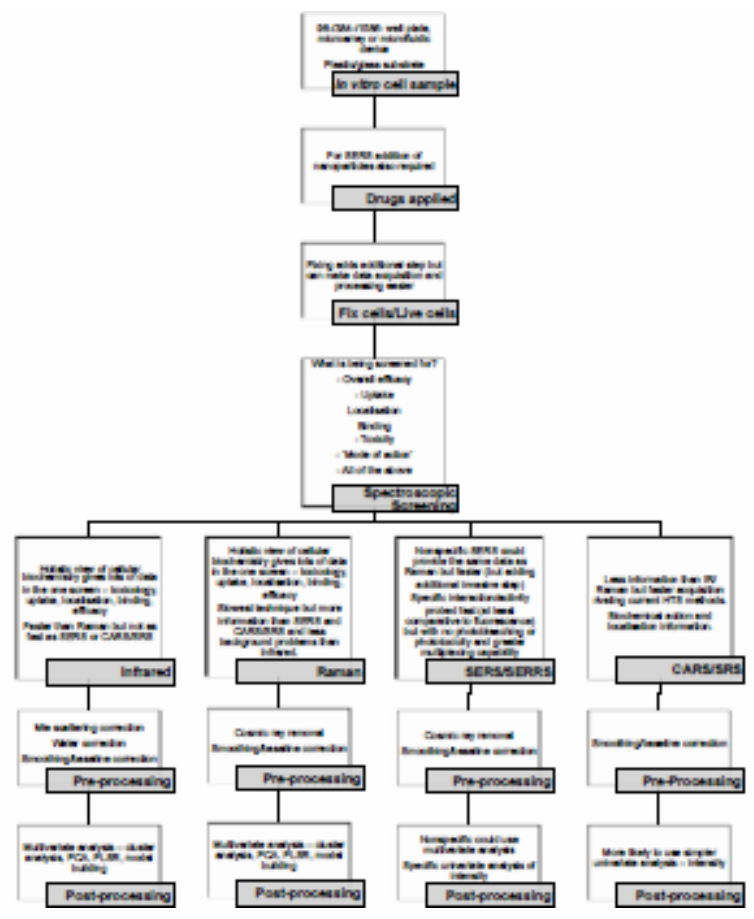

